\title{
Nucleonic gamma-ray production in Vela $X$
}

\author{
D. Horns ${ }^{1}$, F. Aharonian ${ }^{2}$, A. Santangelo ${ }^{1}$, A. I. D. Hoffmann ${ }^{1}$, and C. Masterson ${ }^{2}$ \\ 1 Institute for Astronomy and Astrophysics Tübingen (IAAT), Sand 1, 72076 Tübingen, Germany \\ e-mail: horns@astro.uni-tuebingen.de \\ 2 Max-Planck Institut für Kernphysik (MPIK), PO Box 10 39 80, 69117 Heidelberg, Germany
}

Received 28 February 2006 / Accepted 4 April 2006

\begin{abstract}
Context. The recently discovered extended very high energy gamma-ray $\left(E>0.1 \mathrm{TeV}=10^{11} \mathrm{eV}\right)$ emission from at least three pulsar wind nebula systems (MSH 15-52, G18.0-07, and Vela X) could be hinting at a possibly new class of objects which is called in the following "TeV plerions". In some of these systems, particle acceleration could be driven by ions in the relativistic pulsar wind. These ions are expected to produce gamma-ray emission via inelastic scattering with the ambient medium.

Aims. The gamma-ray emission from the Vela TeV plerion associated with the pulsar PSR B0833-45 is investigated

Methods. We calculate the gamma-ray emission in the framework of nucleonic gamma-ray production including the synchrotron and inverse Compton emission from primary and secondary electrons and compare the model with observational data.

Results. The spectra calculated in this model give a very good description of the gamma-ray data. The required energy in nuclei is $W_{\mathrm{p}}=1.3 \times 10^{49}\left(n / 0.6 \mathrm{~cm}^{-3}\right)^{-1}$ erg for protons and $W_{\mathrm{Fe}} \approx 10^{48} \mathrm{erg}$ for iron nuclei released in the pulsar wind. The secondary electrons produce optical to soft X-ray emission at the level of $\approx 10^{32} \mathrm{erg} / \mathrm{s}$. We give a prediction for the $v_{\mu}$ and $v_{\mathrm{e}}$ neutrino emission including a simple parameterization of the $v_{\mu}$ flux useful for calculating detection rates in next generation neutrino telescopes.

Conclusions. The observed gamma-ray emission can be produced by a pulsar wind which carries a large fraction of the spin down luminosity in the form of relativistic nuclei. We predict the neutrino flux as a unique signature for this model. Based upon this prediction, the Vela $\mathrm{TeV}$ plerion would be one of the best known candidates for detection with next generation neutrino telescopes.
\end{abstract}

Key words. gamma rays: theory - X-rays: general - neutrinos - pulsars: individual: PSR B0833-45 -

radiation mechanisms: non-thermal

\section{Introduction}

The HESS collaboration has recently reported the detection of spatially extended very high energy gamma-ray emission, with integrated luminosity $L_{0.6-65 \mathrm{TeV}} \approx 10^{33} \mathrm{erg} / \mathrm{s}$ (Aharonian et al. 2006), within the extended region of radio emission called "Vela X" (see e.g. Dwarakanath 1991) located in the center of the Vela SNR, and south of the Vela pulsar, PSR B0833-45, which, together with its compact X-ray nebula (Helfand et al. 2001) constitutes a middle-aged, nearby Pulsar Wind Nebula (PWN). PSR B0833-45 is located at a distance of $293_{-17}^{+19} \mathrm{pc}$ (Caraveo et al. 2001; Dodson et al. 2003b) rotating at a frequency $v$ of $11.2 \mathrm{~Hz}$, and slowing down on the average with $\dot{v}=-1.57 \times 10^{-11} \mathrm{~Hz} \mathrm{~s}^{-1}$, which implies a current spin down luminosity of $L_{\mathrm{sd}}=7 \times 10^{36} \mathrm{erg} \mathrm{s}^{-1}$ and a characteristic age of $\tau \approx 11000 \mathrm{yrs}$ (Taylor et al. 1993).

The TeV plerion HESS J0835-455 extends southwest of the Vela pulsar for $\sim 5.7 \mathrm{pc}(F W H M$, at $d=290 \mathrm{pc})$ and measures $4.3 \mathrm{pc}$ across. This is larger than the soft X-ray emission feature noticed by Markwardt \& Ögelman (1995) in ROSAT PSPC data. The ROSAT spectrum, limited to $0.9-2.0 \mathrm{keV}$, appears spatially uniform across the feature and is described either by a thermal plasma model or a power law. Further ASCA observations (0.6-7 keV, Markwardt \& Ögelmann 1997) of the southern tip of this feature alone revealed a spectrum remarkably similar to the rest of the SNR. It is described, when combined with ROSAT by a two temperature plasma model, or by a one temperature plasma model plus power law $\mathrm{d} N_{\mathrm{X}} / \mathrm{d} E \propto E^{-2.1}$ similar to other PWN.
The observed $\mathrm{TeV}$ gamma-ray emission has been interpreted by Aharonian et al. (2006) as inverse Compton emission of nonthermal energetic electrons. The intrinsic electron distribution is constrained by the gamma-ray observations to follow a power law with $\mathrm{d} N_{\mathrm{e}} / \mathrm{d} E \propto E^{-2}$ up to $E \approx 70 \mathrm{TeV}$ with a sharp cut-off at higher energies. The total energy required to match the observations is $W_{\mathrm{e}}=2.2 \times 10^{45} \mathrm{erg}$. This is a surprisingly small fraction $\left(<10^{-3}\right)$ of the total spin down energy of the pulsar which naturally gives rise to the question where the remaining energy of the system could be.

Interestingly, the missing energy could in principle be accounted for if the observed $\mathrm{TeV}$ gamma-rays are produced by hadronic processes, i.e. inelastic interactions of energetic nucleons or nuclei with the ambient interstellar medium $(p N$, $\left.\mathrm{NN} \rightarrow \ldots \pi^{0} \rightarrow 2 \gamma\right)$.

In this Letter, we explore this scenario of nucleonic gammaray production in the Vela $\mathrm{TeV}$ plerion. We show that a coherent model can be developed not only to explain the spectral energy distribution (SED) of the source but also to account for the "missing" spin down energy of the pulsar.

\section{Hadronic gamma-ray production in Vela $X$}

In the magneto-hydrodynamic model of pulsar wind nebulae (Rees \& Gunn 1974; Kennel \& Coroniti 1984), the majority of the spin down power is released in a relativistic wind of particles. Once the particles are isotropized in the vicinity of a relativistic standing shock formed at the interface to the ambient medium, 
electrons and positrons efficiently produce radio to gamma-rays via synchrotron and IC processes. Consistent with this picture the Vela pulsar powers a compact X-ray nebula (Helfand et al. 2001).

It has been suggested that a fraction of the spin down power of pulsars is converted into a wind of nuclei (Hoshino et al. 1992; Arons \& Tavani 1994; Gallant \& Arons 1994) which ultimately excites resonant waves in the downstream region thus giving rise to acceleration of electrons to a power-law type energy spectrum (primary electrons, see also Sect. 3). In this model, the pulsar wind is expected to consist of a mixture of nuclei (e.g. iron and less massive nuclei resulting from photo-dissociation of iron nuclei) and electrons which could also contribute to the overall cosmic ray flux in the Galaxy (Bednarek \& Protheroe 1997) and produce gamma-rays (Amato et al. 2003; Bednarek \& Bartosik 2003) and neutrinos (Bednarek 2003). The importance of hadronic gamma-ray production in the case of the Crab nebula was indicated earlier by Atoyan \& Aharonian (1996).

In the following, we assume that energetic nuclei released by the wind are captured within the $\mathrm{TeV}$ plerion and produce gamma-rays via inelastic scattering on the thermal gas as target material. We estimate here the diffusive escape time $t_{\text {diff }}$, and the time scale for pion production in order to estimate the required energy in nuclei to match the observed gamma-ray luminosity.

The time scale for escape of the nuclei from the TeV plerion with an effective radius $R$ is taken to be the diffusion time scale: $t_{\text {diff }}=R^{2}[2 D(E)]^{-1}$ The effective radius is assumed to be $4 \mathrm{pc}$ taking into account that the position of the pulsar is off-centered. For the diffusion coefficient $D(E)$ a Bohm type diffusion is assumed: $D(E)=\eta r_{\mathrm{G}} c / 3$ with the gyro factor $\eta \geq 1$ (in the Bohm limit $\eta=1)$. Then:

$$
t_{\mathrm{diff}}=7300 \mathrm{yrs} Z \eta^{-1}\left(\frac{R}{4 \mathrm{pc}}\right)^{2} \cdot\left(\frac{B}{10 \mu \mathrm{G}}\right) \cdot\left(\frac{E}{100 \mathrm{TeV}}\right)^{-1},
$$

with $B$ the average magnetic field and $Z$ the charge number of the nuclei. The energy of the nuclei is therefore reduced by $\zeta=$ $\min \left[1, t_{\mathrm{diff}} / \tau\right]$ (for protons $\zeta=0.7$ while for nuclei with $Z>1$, $\zeta=1)$.

The diffusive escape time of nuclei with $Z>1$ exceeds the age of the PWN and we conclude that most of the energy released by the pulsar wind since the birth of the pulsar is still present in the form of energetic particles within the TeV plerion. The magnetic field is taken to be $10 \mu \mathrm{G}$ which is smaller than the equipartition field which we calculate from radio measurements (Dwarakanath 1991; Dodson et al. 2003a) to vary between $B_{\text {eq }}=20-50 \mu \mathrm{G}$. In the case of an inverse Compton origin of the gamma-ray emission, the magnetic field is constrained by $\mathrm{X}$-ray data to be well below this equipartition value. In a very simple scenario without any additional seed photons, the magnetic field would have to be smaller than $3 \mu \mathrm{G}$ in order not to exceed the observed X-ray emission (see also Sect. 3). In the hadronic production scenario, the magnetic field is only loosely constrained to be at least $\approx 10 \mu \mathrm{G}$ (the value chosen here) in order to avoid significant energy losses due to escape of particles (see Eq. (1)).

The rate of inelastic $\mathrm{NN} \rightarrow \ldots \pi^{0}$ interaction depends on the composition of the pulsar wind as well as on the composition and number density $n$ of the target material. The presence of a thermal X-ray emitting plasma is consistent with a plasma density of $0.6 \mathrm{~cm}^{-3}$ (Markwardt \& Ögelman 1995) confirmed with the ASCA data discussed in Sect. 3. This value should be interpreted as a lower limit to the actual density. The composition of the thermal plasma is consistent with solar metallicity while the composition of the pulsar wind is largely unknown.
We note however, that the total cross section for $\mathrm{pN}$ interaction is $\sigma_{\mathrm{pN}} \propto A^{2 / 3} \sigma_{\mathrm{pp}}$ with $A$ the mass number of the nucleus and $\sigma_{\mathrm{pp}}$ the total cross section for pp interactions. Similarly, the cross section for NN-type interactions is greater $\left(\sigma_{\mathrm{NN}}>\sigma_{\mathrm{pN}}\right)$. Furthermore, the inelasticity of pion production $\xi$ decreases only moderately with the mass number. Next, we calculate the average time for $\pi^{0}$ production $t_{\mathrm{pp}}$ only for $\mathrm{pp}$ type interactions, bearing however in mind that the corresponding time for $\mathrm{pN}$ and $\mathrm{NN}$ interactions is smaller than for $\mathrm{pp}$ interactions.

$t_{\mathrm{pp}}=\left(\xi_{\mathrm{pp}} n \sigma_{\mathrm{pp}} c\right)^{-1} \approx 2.5 \times 10^{8} \mathrm{yrs}\left(n / 0.6 \mathrm{~cm}^{-3}\right)^{-1}$

with $\xi_{\mathrm{pp}} \approx 0.15$. We have also calculated the $\pi^{0}$ production time for heavier nuclei interactions by means of Monte Carlo simulations using the QGSJet event generator (Kalmykov et al. 1997). We find that $t_{\mathrm{Fep}} \approx 0.1 t_{\mathrm{pp}}$ for a pulsar wind predominantly loaded with iron nuclei.

Finally, we calculate the total energy in protons $W_{\mathrm{p}}$ required to match the gamma-ray luminosity $W_{\mathrm{p}}=L_{\gamma} \cdot t_{\mathrm{pp}} / \zeta$ :

$W_{\mathrm{p}}=1.1 \times 10^{49} \mathrm{erg}\left(\frac{L_{\gamma}}{10^{33} \mathrm{erg} / \mathrm{s}}\right)\left(\frac{n}{0.6 \mathrm{~cm}^{-3}}\right)^{-1}\left(\frac{\zeta}{0.7}\right)^{-1}$.

For a pulsar wind predominantly loaded with iron nuclei, $W_{\mathrm{N}} \approx$ $10^{48} \mathrm{erg}$. In addition, we have calculated the spectrum of gamma-rays from $\pi^{0}$ decay. The differential gamma-ray flux has been calculated using recently published energy dependent parameterizations of the inelastic total cross section and differential $\pi^{0}$ production for pp interactions (Kelner et al. 2006). The new parameterizations take also the contribution of $\eta^{0}$ decay to the gamma-ray production into account. We note that a more general treatment of heavier nuclei interaction and the resulting differential gamma-ray spectrum is beyond the scope of this Letter. For this calculation, we assume that the differential energy spectrum of the total number of particles is close to a mono-energetic distribution with most particles having an energy of $E_{\mathrm{c}}: \mathrm{d} N_{\mathrm{p}} / \mathrm{d} E=C \cdot E^{2} \exp \left[-\left(E / E_{\mathrm{c}}\right)^{2}\right]$. This energy distribution is expected in the case of thermalization in the downstream region of a relativistic shock with nucleons suffering additional energy losses from the resonant acceleration of pairs. We have adopted a super-exponential cut-off to parameterize the effect of energy losses giving a fairly good description of the downstream energy distribution of ions found in simulations (Arons \& Tavani 1994).

The best fit of the data is obtained with $E_{\mathrm{c}}=80 \mathrm{TeV}$ and a normalization value for $C$ that corresponds to an injected proton energy: $W_{\mathrm{p}}=\zeta^{-1} \int E \cdot \mathrm{d} N_{\mathrm{p}} / \mathrm{d} E \mathrm{~d} E=1.3 \times 10^{49} \mathrm{erg}$. This value is consistent with the estimate for $W_{\mathrm{p}}$ obtained in Eq. (3). The resulting gamma-ray spectrum is shown in Fig. 1.

\subsection{Secondary electrons and neutrino production}

Besides gamma-rays, secondary electrons are produced from pion decays. For the considered magnetic field $B=10 \mu \mathrm{G}$, the secondary electrons lose energy mainly via synchrotron emission. In order to calculate the resulting SED, we assume that the differential energy spectrum of the total number of electrons $N(E)$ has reached a steady state. The kinetic equation which describes the temporal evolution of this spectrum, taking only (the dominant) synchrotron radiation losses into account, is simplified giving thus for $N(E)$ the solution: $N(E)=$ $\frac{1}{E} \int^{E} Q\left(E^{\prime}\right) \mathrm{d} E^{\prime}$ with the energy loss $\dot{E}=-b E^{2}, b=2.55 \times$ $10^{-3} \mathrm{TeV}^{-1} \mathrm{~s}^{-1} \cdot(B / \mathrm{G})^{2}$. The resulting secondary particle spectra in the steady state limit can be considered as upper limits. 


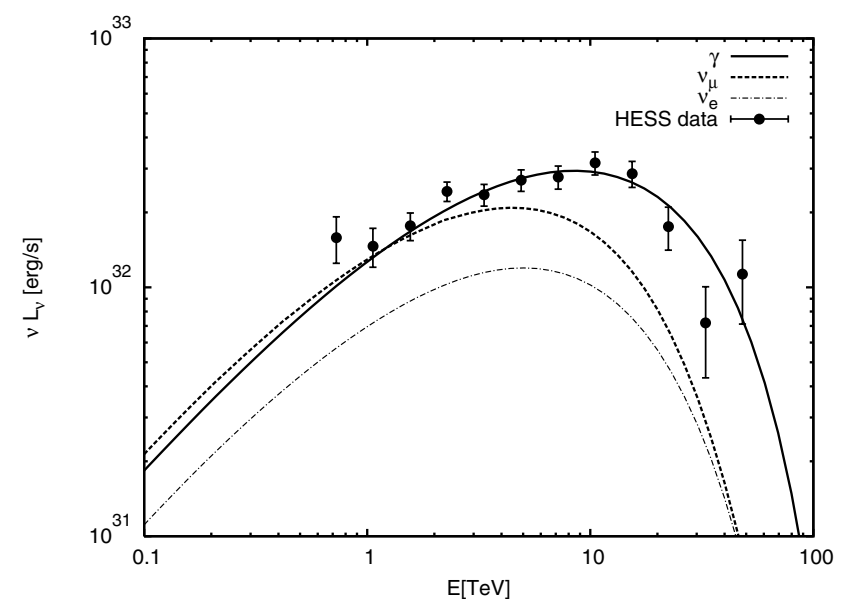

Fig. 1. SED for gamma-ray, $v_{\mu}$, and $v_{\mathrm{e}}$ for the injected proton energy $W_{\mathrm{p}}=1.3 \times 10^{49}$ erg assuming pp interactions with an ambient medium with a density of $0.6 \mathrm{~cm}^{-3}$.

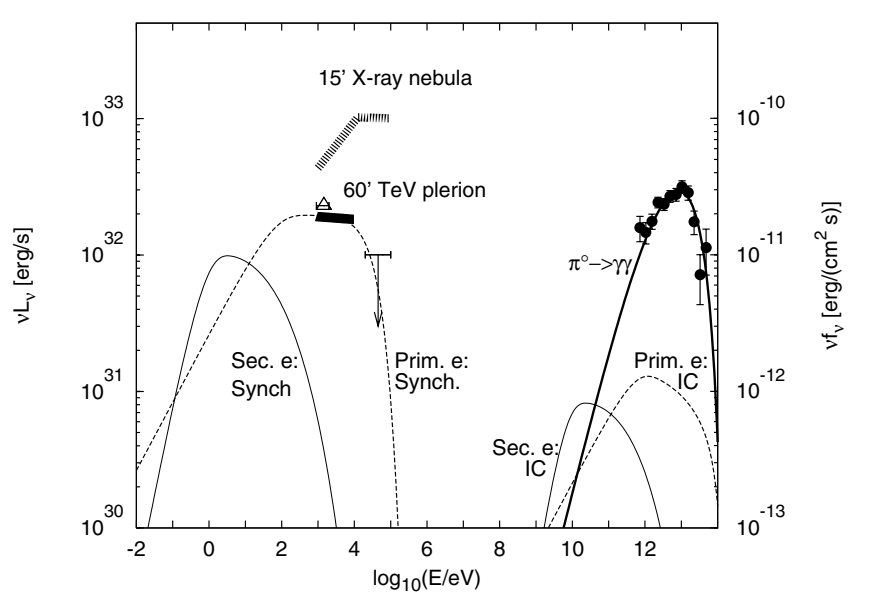

Fig. 2. The overall SED is shown including the gamma-ray emission from $\pi^{0}$-decay and the respective synchrotron and IC emission of primary and secondary electrons in a $10 \mu \mathrm{G}$ field. The extended hard X-ray emission seen with ASCA (solid thick line) from the TeV plerion is matched with a primary electron component $W_{\mathrm{e}}=10^{45} \operatorname{erg}(B / 10 \mu \mathrm{G})^{-2}$. The downward pointing arrow is the $90 \%$ c.l. upper limit from INTEGRAL ISGRI data between $20-60 \mathrm{keV}$ from the TeV plerion. The broad dashed curve indicates the combined XMM-Newton and BeppoSAX spectrum from the compact nebula (Mangano et al. 2005), the open triangle is the ROSAT measurement as given by Markwardt \& Ögelman (1995).

The source term $Q(E)$ is derived from the production rate of electrons in pp interactions (see above).

We have introduced a lower energy cut-off in the steady state solution to take into account that particles with the energy $E_{\mathrm{c}}$ have not had time to cool below an energy $E(\tau, B)=\left(E_{\mathrm{c}}^{-1}+\right.$ $\left.b \tau B^{2}\right)^{-1}$. As an approximation, we use a lower energy cut-off in the electron distribution at $E(t=11 \mathrm{kyrs}, B=10 \mu \mathrm{G})$.

In Fig. 2, the overall spectral energy distribution is shown, including the secondary electron synchrotron emission for a magnetic field of $10 \mu \mathrm{G}$ and the corresponding IC emission with the $\mathrm{CMB}$ as the seed photons. Published searches for optical emission from the Vela PWN have so far only produced upper limits on the surface brightness limited to small $\left(O(10) \operatorname{arcsec}^{2}\right)$ regions (Mignani et al. 2003). A simple scaling of the surface brightness to the TeV plerion results in upper limits $>10^{34} \mathrm{erg} \mathrm{s}^{-1}$, at least two orders of magnitude higher than the predicted secondary electrons' synchrotron emission. The secondary electrons' inverse Compton emission is fainter than the gamma-ray emission from pion decay and not detectable. Also shown in Fig. 2 is the synchrotron and IC emission from a population of primary electrons, as discussed in the next section. Based on the observed gamma-ray emission we predict $v_{\mu}$ (including $\overline{v_{\mu}}$ ) and $v_{\mathrm{e}}$ emission (from $\mu$ decay) from the Vela TeV plerion using the parameterization given by Kelner et al. (2006). The differential 0.1-30 TeV $v_{\mu}$ flux can be expressed with an accuracy of $2 \%$ as

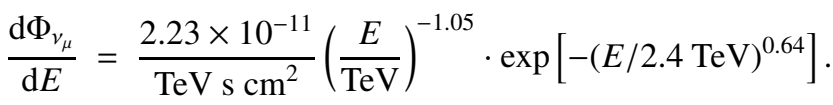

The observable $v_{\mu}$ flux at Earth is reduced by neutrino oscillations not included in Eq. (4) by roughly a factor of 2 (Costantini $\&$ Vissani 2005). The emitted power in $v_{\mu}$ and $v_{\mathrm{e}}$ is shown in Fig. 1 together with the gamma-ray data.

\section{Primary electrons and X-ray emission}

The secondary electrons produced by the inelastic nuclear scattering do not (independent of the magnetic field) explain the observed non-thermal (most likely synchrotron) extended $\left(>15^{\prime}\right)$ emission in the X-ray band (Harnden et al. 1985; Markwardt \& Ögelman 1995; Markwardt \& Ögelmann 1997). X-ray observations of the inner $15^{\prime}$ centered on the Vela Pulsar (Mangano et al. 2005) indicate that electrons injected with a $E^{-2}$ type spectrum cool radiatively to $E^{-3}$ while escaping the compact nebula. Together with the nucleons, these cooled electrons eventually fill at least a fraction of the large $\mathrm{TeV}$ plerion volume. In order to investigate the presence of non-thermal X-rays from the $\mathrm{TeV}$ plerion, we have extracted the energy spectrum from unpublished ASCA GIS2\&3 data (Obs.Id 25038000 for a total of $76 \mathrm{ksec}$ ) covering the central part of the $\mathrm{TeV}$ plerion excluding the bright compact X-ray nebula in the north.

The extraction region (limited by the ASCA GIS field of view) is smaller than the $\mathrm{TeV}$ plerion extension. Using the Gaussian surface brightness profile given in Aharonian et al. (2006), we estimate that $40 \%$ of the total gamma-ray flux is emitted within the region used to extract the X-ray spectrum. The X-ray energy spectrum in the range from 1 to $10 \mathrm{keV}$ is best fit using a model which combines a thermal $(k T=0.3 \mathrm{keV})$ and a non-thermal plasma-component represented by a power law component up to $10 \mathrm{keV}$ with a photon index $\Gamma=2 \pm 0.1_{\text {stat }} \pm$ $0.2_{\text {sys }}$, consistent with the combined ASCA and ROSAT spectrum from the head region of the TeV plerion (Markwardt \& Ögelmann 1997).

The total unabsorbed energy flux of the power law component between 2 and $10 \mathrm{keV}$ is $f_{2-10}=\left(3.3 \pm 0.1_{\text {stat }} \pm 0.6_{\text {sys }}\right) \times$ $10^{-11} \mathrm{erg}\left(\mathrm{cm}^{2} \mathrm{~s}\right)^{-1}$. The energy spectra extracted at various positions within the $\mathrm{TeV}$ plerion do not indicate any systematic differences on the spectral shape. The (90\% c.l.) upper limit derived from INTEGRAL data taken between 20 and $60 \mathrm{keV}$ is well below the extrapolations of the ASCA energy spectrum indicating a cut-off in the energy spectrum.

The observed X-ray flux can be accounted for by the primary electron component released by the pulsar wind in the TeV plerion with a total energy of $W_{\mathrm{e}} \approx 10^{45}(B / 10 \mu \mathrm{G})^{-2}$ erg between $E_{\text {min }}=0.01 \mathrm{TeV}$ and $E_{\max }=200 \mathrm{TeV}$. The injection spectrum follows a power law with $\mathrm{d} N_{\mathrm{e}} / \mathrm{d} E \propto E^{-2}$ which is modified by a cooling break at $E_{\mathrm{b}}=1.0 /\left(b B^{2} \tau\right) \approx 10(B / 10 \mu \mathrm{G})^{-2} \mathrm{TeV}$ to continue with $\mathrm{d} N_{\mathrm{e}} / \mathrm{d} E \propto E^{-3}$ for $E>E_{\mathrm{b}}$. The resulting synchrotron and inverse Compton spectra are shown in Fig. 2. 


\section{Conclusion}

Motivated by the recent HESS detection of extended emission from the Vela $\mathrm{TeV}$ plerion, we have investigated a hadronic production scenario where gamma-rays are produced in inelastic collisions of energetic nuclei released by the pulsar wind. In this scenario, the total energy in the nucleons is required to match the observed gamma-ray emission ranges from $10^{48}-10^{49} \mathrm{erg}$ where the lower value corresponds to an iron and the higher value to a proton loaded wind.

This energy is well balanced by the total spin down energy of the pulsar which ranges from $5 \times 10^{48}-5 \times 10^{51}$ erg depending on the braking index $n_{\mathrm{b}}$ which for the Vela pulsar has been determined to be as small as $n_{\mathrm{b}}=1.4 \pm 0.2$ (Lyne et al. 1996). We find that secondary electrons from the same inelastic interactions responsible for the observed gamma-ray emission produce synchrotron emission in the optical to soft X-ray (depending on the magnetic field strength).

From the analysis of ASCA archival data of the $\mathrm{TeV}$ plerion, a power-law type X-ray spectrum $\left(\mathrm{d} N_{\mathrm{X}} / \mathrm{d} E \propto E^{-2}\right)$ between 1 and $10 \mathrm{keV}$ has been found. When combining the ASCA spectrum with an upper limit from INTEGRAL observations, a sharp cut-off between 10 and $20 \mathrm{keV}$ is implied. The spectrum and flux is well explained by a radiatively cooled primary electron component originating from the pulsar wind with a total energy of $10^{45} \mathrm{erg}$ (again for a $10 \mu \mathrm{G}$ field).

Observations of neutrinos, which can only be expected in case of hadronic origin, are key to discern the mechanism for the production of $\mathrm{TeV}$ gamma-rays. Among currently known $\mathrm{TeV}$ sources, the Vela $\mathrm{TeV}$ plerion appears to be a favorable candidate as its rather hard spectrum (see Fig. 1 and Eq. (4)) would result in high neutrino detection rates comparable or even exceeding the rates estimated for RX J1713-3946 (Costantini \& Vissani 2005).

Acknowledgements. We thank N. Tserendorj for providing results from the ASCA observations. This research has made use of NASA's ADS. AS, AH, and DH are thankful to the University of Tübingen, especially the IAAT, for making this work possible. We thank the referee for the valuable suggestions and comments.

\section{References}

Aharonian, F. A., et al. 2006, A\&A, 438, L43

Amato, E., Guetta, D., \& Blasi, P. 2003, A\&A, 402, 827

Arons, J., \& Tavani, M. 1994, ApJS, 90, 797

Atoyan, A. M., \& Aharonian, F. A. 1996, MNRAS, 278, 525

Bednarek, W., \& Protheroe, R. J. 1997, PRL, 79, 2616

Bednarek, W. 2003, A\&A, 407, 1

Bednarek, W., \& Bartosik, M. 2003, A\&A, 405, 689

Caraveo, P. A., De Luca, A., Mignani, R. P., \& Bignami, G. F. 2001, ApJ, 561, 930

Costantini, M. L., \& Vissani, F. 2005, Astropart. Phys. 23, 477

Dodson, R., Lewis, D., McConnell, D., \& Deshpande, A. A. 2003a, MNRAS, 343, 116

Dodson, R., Legge, D., Reynolds, J. E., \& McCulloch, P. M. 2003b, ApJ, 596, 1137

Dwarakanath, K. S. 1991, JApA, 12, 199

Gallant, Y. A., \& Arons, J. 1994, ApJ, 435, 230

Harnden, F. R., Grant, P. D., Seward, F. D., \& Kahn, S. M. 1985, ApJ, 299, 828

Helfand, D. J., Gotthelf, E. V., \& Halpern, J. P. 2001, ApJ, 556, 380

Hoshino, M., Arons, J., Gallant, Y. A., \& Langdon, A. B. 1992, ApJ, 390, 454

Kalmykov, N. N., Ostapchenko, S. S., \& Pavlov, A. I. 1997, Nucl. Phys. Proc. Suppl., B52, 17

Kelner, S. R., Aharonian, F. A., \& Bugayov, V. V. 2006, Phys. Rev. D, submitted Kennel, C. F., \& Coroniti, F. V. 1984, ApJ, 283, 694

Lyne, A. G., Pritchard, R. S., Graham-Smith, F., \& Camilo, F. 1996, Nature, 381, 497

Mangano, V., Massaro, E., Bocchino, F., Mineo, T., \& Cusumano, G. 2005, A\&A, 436, 917

Markwardt, C. B., \& Ögelman, H. 1995, Nature, 375, 40

Markwardt, C. B., \& Ögelman, H. B. 1997, ApJ, 480, L13

Mignani, R. P., De Luca, A., Kargaltsev, O., et al. 2003, ApJ, 594, 419

Milne, D. K. 1995, MNRAS, 277, 1435

Rees, M. J., \& Gunn, J. E. 1974, MNRAS, 167, 1

Taylor, J. H., Manchester, R. N., \& Lyne, A. G. 1993, ApJS, 88, 529 